\title{
Laparo-endoscopic single site hysterectomy in renal transplant women using conventional laparoscopic instruments
}

\author{
Wei-An Goh ${ }^{1}$, Eunice MX Tan ${ }^{1}$, Ravichandran Nadarajah ${ }^{2}$ \\ ${ }^{1}$ Yong Loo Lin School of Medicine, National University of Singapore, Singapore 117597, Singapore. \\ ${ }^{2}$ Department of Obstetrics and Gynaecology, SingHealth, Singapore 169608, Singapore.
}

Correspondence to: Dr. Ravichandran Nadarajah, Department of Obstetrics and Gynaecology, SingHealth, Outram Rd, Singapore 169608, Singapore. E-mail: ravichandran.nadarajah@singhealth.com.sg

\begin{abstract}
How to cite this article: Goh WA, Tan EM, Nadarajah R. Laparo-endoscopic single site hysterectomy in renal transplant women using conventional laparoscopic instruments. Mini-invasive Surg 2021;5:30. https://dx.doi.org/10.20517/2574-1225.2021.42
\end{abstract}

Received: 22 Mar 2021 First Decision: 26 Apr 2021 Revised: 5 May 2021 Accepted: 12 May 2021 First online: 27 May 2021

Academic Editor: Simone Ferrero Copy Editor: Xi-Jun Chen Production Editor: Xi-Jun Chen

\begin{abstract}
Kidney transplant recipients are at a higher risk of developing cancers as compared to the general population. This is of concern when it comes to gynaecological pathologies because the transplanted kidney lies in the pelvic region, in close proximity to the diseased organ. The successful use of laparo-endoscopic single site surgery with conventional laparoscopic instruments for total hysterectomy and bilateral salpingo-oophorectomy in three patients with prior renal transplantation is reported.
\end{abstract}

Keywords: Laparo-endoscopic single site surgery, hysterectomy, renal transplant, laparoscopy, ovarian tumour, endometrial cancer

\section{INTRODUCTION}

The prevalence and incidence of end-stage renal disease (ESRD) has been increasing across the world. It is expected that around $1.5 \%$ of people with an estimated glomerular filtration rate of $15-60 \mathrm{~mL} / \mathrm{min} / 1.73 \mathrm{~m}^{2}$ transition to ESRD every year ${ }^{[1]}$. The standard of care for patients with ESRD is either dialysis or kidney transplantation. Numerous studies have shown that patient survival is significantly higher with kidney transplantation than with dialysis. Moreover, with the use of better immunosuppression and improved surgical techniques, graft and patient survival after kidney transplantation have improved over the years ${ }^{[2,3]}$. 
However, it is known that incidence of cancer is higher in transplant recipients than in patients undergoing dialysis for ESRD. It has been hypothesised that this could be attributed to the immunosuppressants regime transplant recipients received ${ }^{[4]}$.

This case report describes three cases of gynaecologic pathologies in kidney transplant recipients and the use of laparo-endoscopic single site surgery (LESS) to perform a total hysterectomy and bilateral salpingooophorectomy (THBSO). In our literature review, only Zhang and $\mathrm{Li}^{[5]}$ previously described the use of LESS for THBSO in kidney transplant recipients, but there was minimal information on the type of instruments used and the management of these patients perioperatively. Therefore, to our best knowledge, performing LESS using homemade gloves and conventional laparoscopic instruments in renal transplant recipients has never been described before.

\section{CASE REPORT}

\section{Case \# 1}

A 61-year-old female with a body mass index (BMI) of 22.5, who had one child delivered through normal vaginal delivery, presented with significant post-menopausal bleeding of one week duration requiring admission and blood transfusion in view of her low haemoglobin count which dropped from 12.0 to $7.7 \mathrm{~g} / \mathrm{dL}$. She has a significant past medical history of hypertension, hyperlipidaemia, ESRD secondary to hypertensive nephrosclerosis, mitral regurgitation and atrial fibrillation. She eventually had a deceased donor renal transplantation to the right iliac fossa [Figure 1A]. Her immunosuppressants were $5 \mathrm{mg}$ oral prednisolone and $100 \mathrm{mg}$ oral ciclosporin. She was not initiated on anti-coagulants for her recently diagnosed atrial fibrillation in view of her upcoming gynaecological surgery.

On her abdomen, there was a suprapubic transverse scar extending to the right iliac fossa. The right transplanted kidney was palpable over the right iliac fossa. On speculum, vagina and bimanual examination, the cervix appeared normal, and no obvious mass was felt.

A pelvic ultrasound was performed, which revealed a thickened endometrium of $5.7 \mathrm{~mm}$, a $25 \mathrm{~mm} \times 17 \mathrm{~mm}$ $\times 23 \mathrm{~mm}$ unilocular mass in the right adnexal region with no fluid in the Pouch of Douglas and no other masses seen in the pelvis. The IOTA score was $20 \%$. Subsequently, histological features obtained from an endometrial biopsy suggested anovulatory cycles with disordered proliferation. At the same time, a pelvic MRI [Figure 1B] was ordered, but the malignant potential of the ovarian mass was still uncertain. Her CA125 level was 57.9. Her Papanicolaou smear yielded no abnormal finding. In view of the significant postmenopausal bleeding in the presence of a right adnexal mass, our impression was an ovarian hormone secreting tumour. Treatment options were discussed with the patient; she was keen for surgery and counselled for LESS THBSO and omental biopsy.

During the surgery, a homemade single-port system comprising of the Alexis ${ }^{\bullet}$ wound retractor (Applied Medical, CA, USA) and a $7 \frac{1}{2}$ surgical glove was used. After making a $2.5 \mathrm{~cm}$ umbilical skin incision, the wound retractor was inserted into the peritoneal cavity through the umbilicus, and the glove was fixed to the outer ring of the wound retractor. Upon making small incisions in the fingertip portions of the glove, two 5-mm trocars and one 12- $\mathrm{mm}$ trocar were inserted. A rigid $30^{\circ}, 5 \mathrm{~mm}$ diameter, $45 \mathrm{~cm}$ long endoscope was used. The transplanted kidney was visualised at the right iliac fossa. A diagnostic laparoscopy established slightly enlarged bilateral ovarian masses without any evidence of ovarian malignancy. The right ovary measured $3.2 \mathrm{~cm} \times 2 \mathrm{~cm} \times 1.6 \mathrm{~cm}$, while the left ovary was $4 \mathrm{~cm} \times 1.5 \mathrm{~cm} \times 1.5 \mathrm{~cm}$. Overall, the procedure was uneventful with minimal blood loss. The final histopathological diagnosis for the patient turned out to be disordered proliferative endometrium and bilateral ovarian Sertoli-Leydig cell tumour 

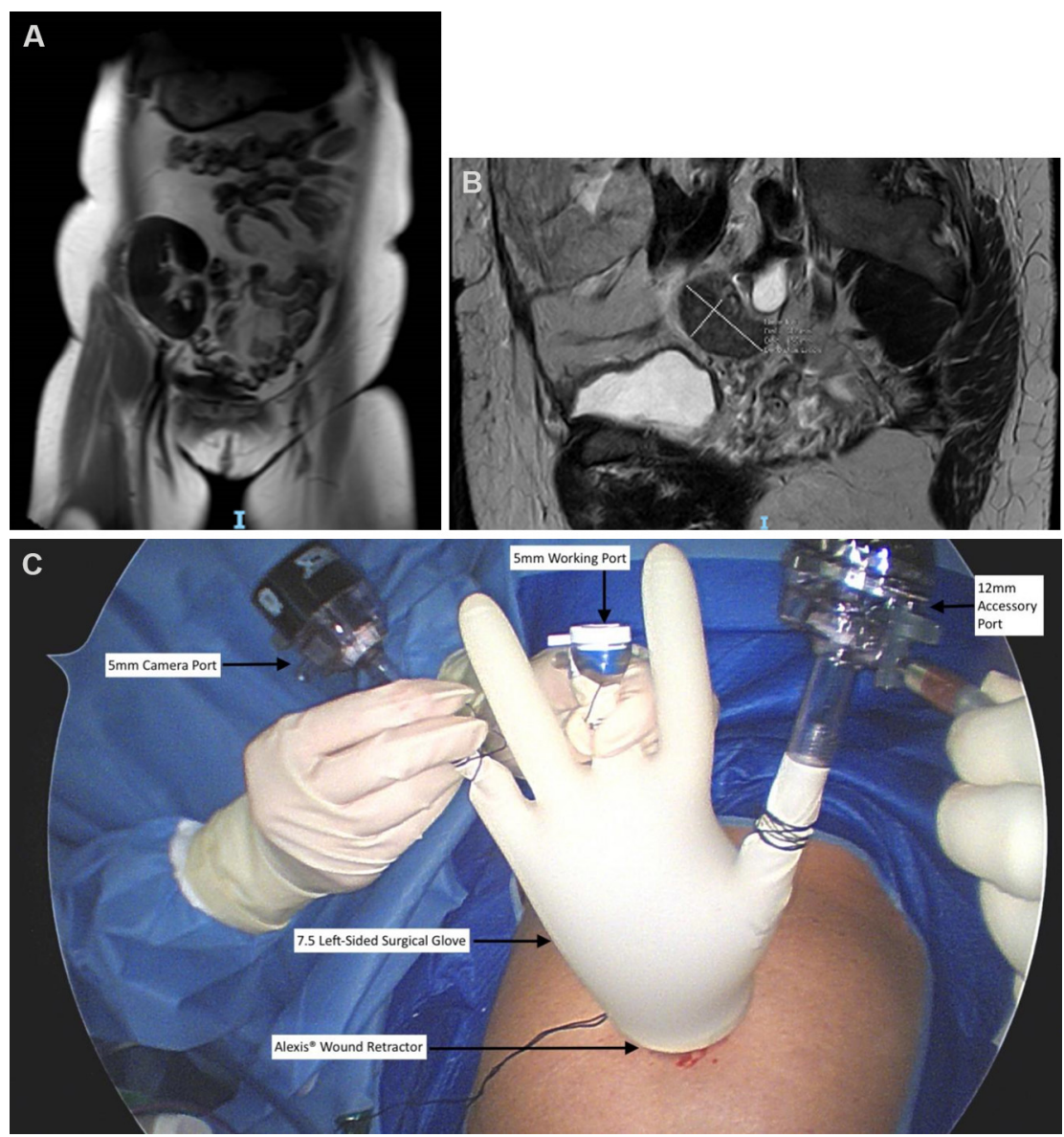

Figure 1. Pelvic MRI showing the transplanted kidney in the right iliac fossa (A). Pelvic MRI showing the right adnexal mass, measuring $25 \mathrm{~mm} \times 17 \mathrm{~mm} \times 23 \mathrm{~mm}$ (B). Labelled photo of the homemade single-port system (C).

suggestive of a benign lesion.

Postoperatively, the patient recovered well. Her renal function was monitored closely together with the renal transplant physician, and she was discharged 2 days later with a 6-day antibiotic course of $1800 \mathrm{mg}$ oral clindamycin.

\section{Case \# 2}

Our second patient was a 49-year-old female with a BMI of 33.2 who is married with three children. She was referred for heavy menstrual bleeding with no anaemic symptoms. Her menstrual cycles were regular, lasting 4-5 days each with heavy flow and formation of multiple small clots. Her significant past medical history includes ESRD secondary to chronic tubulointerstitial nephritis, hyperparathyroidism and posttransplant diabetes mellitus. She had previous surgeries for one termination of pregnancy, one lower segment Caesarean section, two normal vaginal deliveries, tubal ligation and two deceased donor renal transplants with both transplanted kidneys sited at the right iliac fossa [Figure 2A]. Her immunosuppressant regime was $3.5 \mathrm{mg}$ everolimus, $3 \mathrm{mg}$ oral tacrolimus and $5 \mathrm{mg}$ oral prednisolone.

On examination, there was a suprapubic transverse scar extending to the right iliac fossa. The transplanted kidney was not palpable due to her high BMI. On speculum, vaginal and bimanual examination, the cervix was normal, and no obvious mass was felt. 

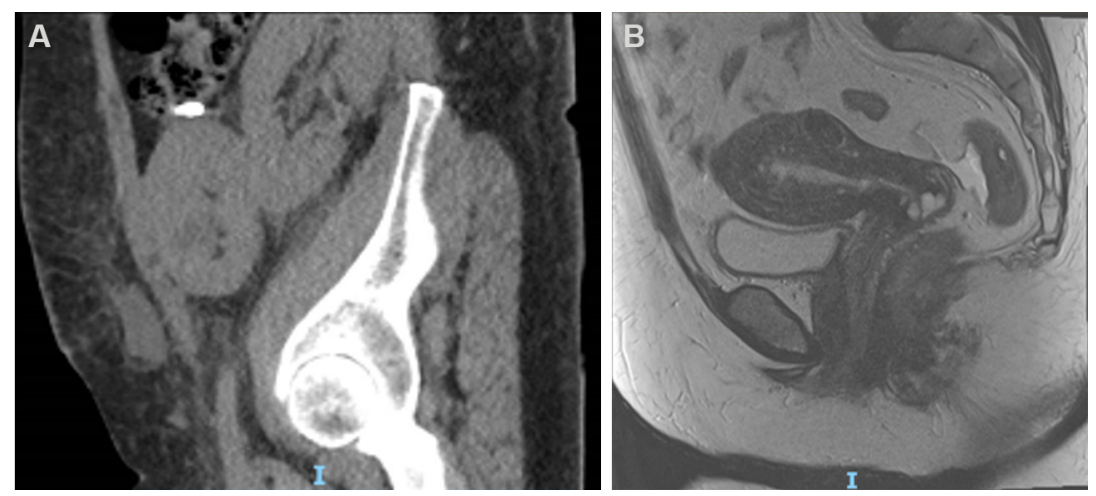

Figure 2. Pelvic sagittal view CT showing the dual kidney transplant taken immediately after the transplantation surgery (A). Pelvic sagittal view MRI showing endometrial lesion confined to the uterus (B).

A pelvic ultrasound was performed, revealing an endometrial thickness of $16.7 \mathrm{~mm}$, two subserosal fibroids of 2.3 and $1.9 \mathrm{~mm}$, multiple non-suspicious cysts in the left and right adnexa and a 2-cm polyp arising from the upper uterine cavity. A hysteroscopy was done for polypectomy and dilatation and curettage. The histological results of the polyp were suggestive of Grade 1 endometrioid carcinoma.

Subsequently, a pelvic MRI and thoracic and abdominal CT were ordered. The MRI results show that the cancer was confined to the endometrium without any myometrial extension or enlarged pelvic lymph nodes [Figure $2 \mathrm{~B}$ ]. Similarly, the CT scan revealed no significant enlarged lymph node elsewhere to suggest distant metastases. She was counselled for THBSO without pelvic lymph nodes dissection as it was a Grade 1 endometrioid carcinoma and, radiologically, the tumour was found to be confined to the endometrium.

The surgical technique was similar to the first case described above. However, in view of her habitus, 45-cmlong bariatric laparoscopic instruments and a 50-cm endoscope were used. The transplanted kidney was visualised at the right iliac fossa. The uterus and bilateral ovaries were normal. The procedure was uneventful and there was minimal bleeding. The final histology showed a Stage 1A Grade 1 endometrioid carcinoma of the endometrium, and there was no evidence of residual tumour.

Three days later, the patient developed thrombophlebitis which resolved with antibiotics. The patient was discharged well on Postoperative Day 6.

After discussing with our tumour board, the recommendation was to observe for disease recurrence. Currently, the patient is on follow-up with us and has been under remission for 2 years.

\section{Case \# 3}

The third patient was a 78 -year-old female with a BMI of 18.5 who is married with one child. She was referred for an incidental finding of a $5.5 \mathrm{~cm} \times 3.6 \mathrm{~cm}$ left adnexal lesion with central hypodensity on pelvic CT initially performed for low back pain [Figure $3 \mathrm{~A}$ ]. She did not complain of any abdominal pain or postmenopausal bleed. Her significant past medical history includes ESRD secondary to chronic glomerulonephritis and recurrent bilateral lower limb deep vein thrombosis. She had a previous living donor kidney transplantation, open myomectomy and laparoscopic cholecystectomy. Her current medications include $8 \mathrm{mg}$ prednisolone, $25 \mathrm{mg}$ azathioprine, $75 \mathrm{mg}$ dipyridamole and $2 \mathrm{mg}$ warfarin. 

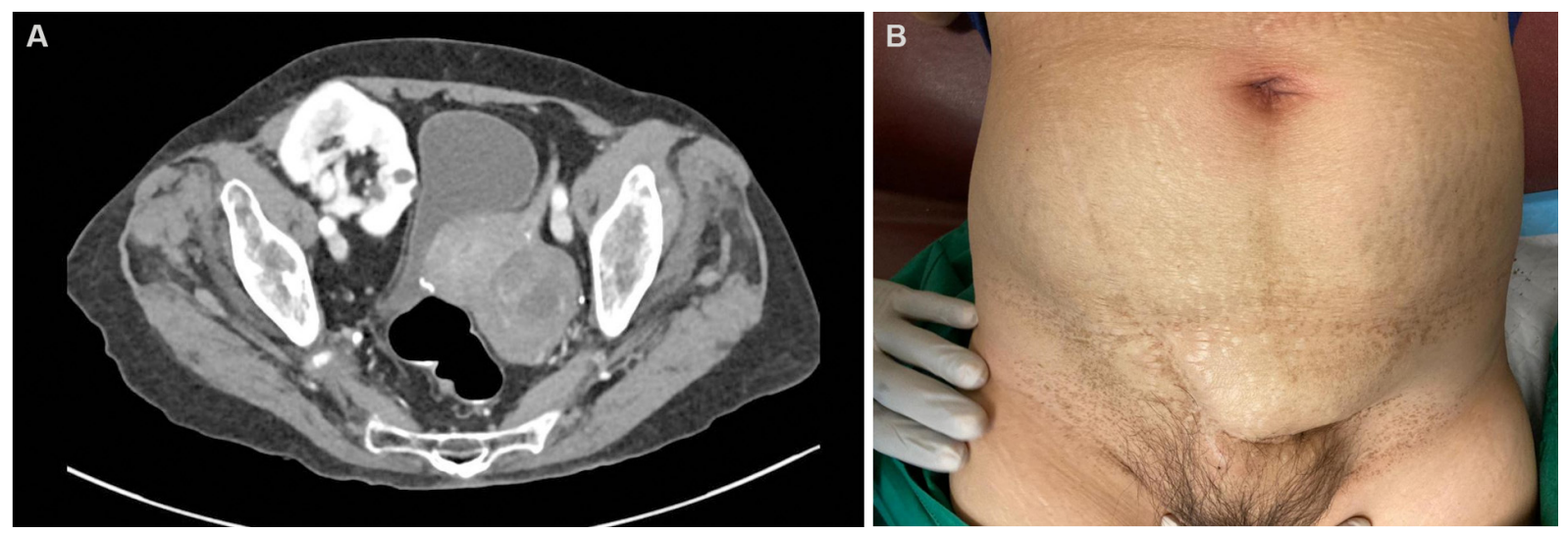

Figure 3. CT Pelvis Axial View showing the left adnexal mass and the transplanted kidney in the right iliac fossa (A). PAbdomen showing suprapubic transverse scar extending to the right iliac fossa (B).

On examination, there was a suprapubic transverse scar extending to the right iliac fossa [Figure 3B]. The right transplanted kidney was palpable over the right iliac fossa. The cervix appeared normal, and no obvious mass was felt on bimanual examination.

Pelvic ultrasound revealed a $6.0 \mathrm{~cm} \times 5.0 \mathrm{~cm} \times 3.5 \mathrm{~cm}$ multiloculated cystic mass in the left adnexal region. Colour Doppler showed mild vascularity within the mass. No ascites or other masses were noted. Tumour markers CA125 and CEA were raised to 71.8 and 11.6, respectively. The IOTA and RMI score were 6.8\% and 646 , respectively, suggesting a high risk of malignancy.

In view of the risk of malignancy and her immunosuppressed state, LESS THBSO was advised, and the patient was keen for the surgery.

Preoperatively, warfarin and dipyridamole were stopped 5 and 2 days prior to surgery, respectively. PT was 10.5, aPTT was 28.2 and INR was 1.0. Her preoperative $\mathrm{Hb}$ was 11.2. The surgical technique was similar to the first case as described above. The procedure was uneventful and there was minimal bleeding $(20 \mathrm{~mL})$. The final histology reported a left ovarian mucinous cystadenoma.

Postoperatively, the patient recovered well and was started on clexane by the haematologist. She was discharged on Postoperative Day 3. Currently, she is on follow-up with the gynaecological oncologist.

\section{DISCUSSION}

Performing a hysterectomy with bilateral salpingo-oophorectomy on a renal transplant recipient is not without its challenge. In a paper written by Heisler regarding hysterectomy in women who have undergone renal transplantation, it is reported that $41.4 \%(n=58)$ of these patients experience postoperative complications, the most common being wound infection and bleeding requiring blood transfusion. Such complications were seen mainly in open hysterectomy ${ }^{[6]}$. This was much higher in comparison to healthy women, in whom the figures are at an estimated $3 \%-22 \%$. In the following paragraphs, we elaborate on the unique challenges faced during the operative care of kidney transplant recipients.

Bleeding requiring blood transfusion was reported as a common complication of hysterectomy in renal transplant recipients. This was because open hysterectomy was performed and many of these patients were on antithrombotic therapy ${ }^{[6]}$. In our first and second cases, the patients were not on these medications. In 
the last case, even though the patient was taking anti-coagulants and anti-thrombotics, intraoperative bleeding was minimal $(100 \mathrm{cc})$, possibly reduced by the use of a laparoscopic approach.

Advances in operative techniques were also able to reduce the risk of intraoperative and postoperative complications in these group of patients. Previously, open laparotomy was the standard approach to performing hysterectomy. However, in recent years, laparoscopy became the more popular choice. Advantages of laparoscopy over laparotomy include shorter hospital stay, decreased adhesion formation and reduced incidence of fever, wound infection, urinary tract infection and pneumonia ${ }^{[7,8]}$. This is especially beneficial in renal transplant recipients because infection is the most common complication of abdominal hysterectomy ${ }^{[6]}$. While it was reported that the incidence of bladder injury is higher in laparoscopic hysterectomies, this risk can be mitigated when it is performed by an experienced surgeon ${ }^{[8]}$.

There are multiple advantages to performing LESS. It leads to a decrease in the risk of visceral and vascular injury related to multiple incisions and trocar placements. Furthermore, the umbilical incision for port placement in LESS is hardly noticeable after healing and offers superior cosmetic results. However, we acknowledge that this may be less important in renal transplant recipients since they already have a large kidney transplant scar. As there is only one entry site, there is also a reduction in postoperative wound infection, hernia formation and elimination of multiple trocar site closures. In addition, LESS is associated with good pain control and lower analgesic requirements, which in turn enhances the recovery of patients $^{[9,10]}$. Overall, LESS has been shown to reduce operative and perioperative complications.

Our surgeon also used a homemade single-port system which accommodates the insertion of various types and sizes of laparoscopic devices, while the elasticity of the glove finger facilitates the retrieval of specimens. The greatest advantage of a homemade port is its cost effectiveness for the patient, as standard, instead of articulated, instruments are utilised. This is feasible because the elasticity of the homemade port coupled with the thin, stretchable umbilical fascia maintains the triangulation, ease of manoeuvre and coordination of the instruments.

A technically challenging aspect of performing hysterectomy in renal transplant patients is to avoid damage to the allograft organ intraoperatively since the surgical field is in the pelvic region where the transplanted kidney is sited. Using LESS is safe because the trocar is only placed at the umbilicus, while a conventional laparoscopy requires a trocar to be inserted at the ipsilateral lower quadrant of the allograft organ, risking injury to it. Although the risk can be reduced in a conventional laparoscopy by placing the trocar more medially in such instances, attention has to be given to identify the inferior epigastric vessels before placing this port ${ }^{[3]}$. Similarly, placing the trocar at a higher position may make the surgery less ergonomic for the surgeon. This renders a single, umbilical port placement safer and more ideal.

Moreover, there is a risk of injury to the urinary bladder during laparoscopic gynaecological surgery, attributed to either the entry process (e.g., suprapubic port insertion) or due to its close proximity with the operating field (e.g., hysterectomy). A previous meta-analysis reported that bladder injury rates range from $0.02 \%$ to $8.3 \%$, making it the most common viscera damaged in conventional laparoscopic pelvic surgery ${ }^{[11]}$. Renal transplant recipients undergoing conventional laparoscopy may have an even higher risk of bladder injury. During renal transplantation, the bladder is lifted supero-anteriorly to allow transplantation of the ureter to the dome of the bladder. Thus, suprapubic port insertion carries an increased risk of bladder injury due to its relatively higher position. While high port placement at the level of or above the umbilicus can be used in place of a suprapubic port, such placements are less ergonomic for the surgeon. On the other hand, entering the peritoneal cavity through a single umbilical incision in LESS minimises the risk of such port- 
related bladder injury.

Retroperitoneal single-port laparoscopic hysterectomies (SP-rH) have been performed to identify the ureter and internal iliac artery, followed by ligation of the uterine artery where it originates from the internal iliac artery. SP-rH can result in less intraoperative bleeding and a decreased risk of ureteral damage ${ }^{[12]}$. However, in patients with renal transplantation, the retroperitoneal approach can be challenging due to anatomical distortions. Furthermore, the major blood vessel supplying the kidney is derived from the external iliac vessels, and inadvertent injury to these vessels using the retroperitoneal approach may affect the function of the allograft kidney. At the same time, identifying the ureter of the allograft kidney may have limited benefit as it is transplanted to the dome of the bladder which is away from the surgical site.

Another obstacle is the high BMI of our second patient in addition to possible adhesions from her previous Caesarean section. Such a patient profile may make LESS more technically challenging and less attractive to surgeons. Some potential issues during the procedure include visual limitations and difficulty in obtaining good triangulation without collisions between instruments. However, in our experience, LESS can still be effectively performed. To improve mobility during the procedure, our surgeon used bariatric laparoscopic instruments and a 50-cm telescope. Additional surgical steps were also performed as the floppy bladder obstructed the view of the vaginal wall. Our surgeon used prolene 2-0 to suspend the uterovesical fold to the anterior abdominal wall in order to lift up the bladder. As a result, the vaginal wall can be easily visualised, which helps facilitate closure of the vaginal wall.

In conclusion, performing a LESS THBSO in renal transplant recipients using conventional laparoscopic instruments provides safe and effective outcomes which are comparable to conventional laparoscopy in the case reports described. There can be clear advantages to performing LESS when appropriate cases are selected, when it is performed by a surgeon experienced in minimally invasive surgery and when a multidisciplinary team is involved in optimising the care of the patient.

\section{DECLARATIONS}

\section{Authors' contributions}

Performed data acquisition and writing of report: Goh WA, Tan EMX

Made contributions to conception of the study, editing and review of the report: Nadarajah $\mathrm{R}$

\section{Availability of data and materials}

Not applicable.

\section{Financial support and sponsorship}

None.

\section{Conflicts of interest}

All authors declared that there are no conflicts of interest.

\section{Ethical approval and consent to participate}

Not applicable.

\section{Consent for publication}

A written informed consent for publication was obtained from all participants. 


\section{Copyright}

(C) The Author(s) 2021.

\section{REFERENCES}

1. Hsu CY, Vittinghoff E, Lin F, Shlipak MG. The incidence of end-stage renal disease is increasing faster than the prevalence of chronic renal insufficiency. Ann Intern Med 2004;141:95-101. DOI PubMed

2. Hart A, Smith JM, Skeans MA, et al. OPTN/SRTR 2016 Annual Data Report: Kidney. Am J Transplant 2018;18 Suppl 1:18-113. DOI PubMed PMC

3. Raff GJ, Kasper KM, Hollinger EF Jr, Goggins WC. Laparoscopic hysterectomy in patients with prior renal transplantation. J Minim Invasive Gynecol 2008;15:223-6. DOI PubMed

4. Kakuda M, Kobayashi E, Tanaka Y, Ueda Y, Yoshino K, Kimura T. Total laparoscopic hysterectomy for endometrial cancer in a renal transplantation patient receiving peritoneal dialysis: Case report and literature review. J Obstet Gynaecol Res 2017;43:1232-7. DOI PubMed

5. Zhang Z, Li M. Single-incision laparoscopic surgery: broaden the implication of minimally invasive surgery to women with prior renal transplant. J Minim Invasive Gynecol 2013;20:S149. DOI

6. Heisler CA, Casiano ER, Gebhart JB. Hysterectomy and perioperative morbidity in women who have undergone renal transplantation. Am J Obstet Gynecol 2010;202:314.e1-4. DOI PubMed

7. Chapron C, Fauconnier A, Goffinet F, Bréart G, Dubuisson JB. Laparoscopic surgery is not inherently dangerous for patients presenting with benign gynaecologic pathology. Results of a meta-analysis. Hum Reprod 2002;17:1334-42. DOI PubMed

8. Aboulfotouh ME, Chaalan F, Mohammed AF. Laparoscopic hysterectomy versus total abdominal hysterectomy: a retrospective study at a tertiary hospital. Gynecol Surg 2020;17:1. DOI

9. Fagotti A, Bottoni C, Vizzielli G, et al. Postoperative pain after conventional laparoscopy and laparoendoscopic single site surgery (LESS) for benign adnexal disease: a randomized trial. Fertil Steril 2011;96:255-9.e2. DOI PubMed

10. Yim GW, Jung YW, Paek J, et al. Transumbilical single-port access versus conventional total laparoscopic hysterectomy: surgical outcomes. Am J Obstet Gynecol 2010;203:26.e1-6. DOI PubMed

11. Minas V, Gul N, Aust T, Doyle M, Rowlands D. Urinary tract injuries in laparoscopic gynaecological surgery; prevention, recognition and management. Obstet Gynaecol 2014;16:19-28. DOI

12. Kim TH, Kim CJ, Kim TJ, et al. Retroperitoneal approach in single-port laparoscopic hysterectomy. JSLS 2016;20:e2016. DOI PubMed PMC 